\title{
Croquages de langue en formation d'enseignants
}

Annemarie Dinvaut, Laboratoire ICTT (Identité Culturelle, Textes \& Théâtralité), Université d'Avignon et des Pays du Vaucluse, France

\author{
VLADIMIR : Nous avons nos raisons. \\ ESTRAGON : Toutes les voix mortes. \\ VLADIMIR : Ça fait un bruit d'ailes. \\ ESTRAGON : De feuilles. \\ VLADIMIR : De sable. \\ ESTRAGON : De feuilles. [...] \\ VLADIMIR : Que disent-elles? \\ ESTRAGON : Elles parlent de leur vie. \\ (Beckett, 1952)
}

Le cursus des futurs enseignants généralistes n'inclut pas nécessairement l'étude volontaire et approfondie d'une langue autre que le français. Par ailleurs, la didactique du français langue seconde ou d'une langue étrangère met souvent en lumière une relation complexe à leur propre biographie langagière, une conscience réduite des langues présentes dans l'environnement et des enjeux des pratiques plurilingues. C'est pourquoi les étudiants en Master 1 des Métiers de l'Enseignement Scolaire, de la Formation et de la Culture (MESFC, Université Lyon 1) participent à un module qui a pour objectif l'introduction à la didactique des langues-cultures et le développement de compétences plurilingues. Nous recourons dans cette formation à une approche pluri-sensorielle et à l'expression plastique. Nous analysons ici ce dispositif et nous interrogeons sur sa pertinence.

\section{1- Le contexte de formation}

Le contexte est celui de l'Unité d'Enseignement «Communication et Culture », qui réfère aux savoirs scientifiques, aux arts visuels, aux langues et cultures. La conception de cette unité d'enseignement et de formation part de plusieurs constats: le manque de confiance en soi pour la pratique de la langue cible et l'enseignement des langues, la difficulté à transférer les compétences d'une langue à l'autre, la méconnaissance, voire le déni, du plurilinguisme ambiant. Le sensoriel apparait dans les recueils des représentations des étudiants sur les langues, mais il est peu pris en compte dans le champ de la didactique : étudiants et enseignants en formation continue concentrent leur énergie sur la recherche du sens (la signification des énoncés linguistiques), au détriment des cinq sens (les perceptions de la matière-langue). En outre, la plupart des ressources didactiques, y compris celles qui 
misent sur des situations d'immersion ou sur la reproduction des conditions d'acquisition de la langue maternelle, font peu de cas de la part du sensoriel dans les apprentissages.

Compte-tenu de ces éléments, l'équipe pédagogique du Master MESFC de Lyon 1 s'est donné comme objectifs de formation le développement d'une conscience et d'une compétence plurilingues. Nous mettons en place une démarche d'éveil aux langues et aux cultures, qui inclut une micro-enquête sociolinguistique et le recueil d'éléments de biographie langagière. Un article sur le paysage sociolinguistique lyonnais (Akinci) et un autre sur les recueils de biographie langagière (Perea et Piccardo) constituent le point de départ à des débats sur le plurilinguisme et les langues fréquentées par les étudiants et à l'élaboration de questionnaires pour une enquête sociolinguistique (détaillée ci-dessous). Outre cette enquête, ils sont invités à faire une collecte de langues, à noter celles qu'ils entendent dans leur environnement proche, ainsi que des indices sur ces langues et les situations de communication (locuteurs, lieu, objet de l'échange) ; ces activités ont déjà été mises en place dans un dispositif de formation antérieur (Dinvaut 345). Les deux éléments nouveaux sont leur extension à tous les groupes d'étudiants, et l'articulation explicite entre les activités : l'enquête, la collecte de langues, la biographie langagière, pour 194 étudiants, et en sus le «croquage de langues » pour 88 d'entre eux. La biographie langagière se fait en deux étapes, via des échanges pendant les Travaux Dirigés et via la production individuelle du Passeport de langues Europass ${ }^{1}$.

Nous faisons l'hypothèse que l'expression plastique peut contribuer positivement à cette démarche de formation et offrir au futur enseignant des pistes pour ses propres pratiques d'enseignement. Pour cela, nous avons demandé à une partie des étudiants de créer pour une langue de leur choix un carnet d'écoute pluri-sensoriel, qui rende visibles leurs perceptions et leurs émotions. Les étudiants reçoivent la consigne suivante: fabriquer un «carnet d'écoutage de langue », sur le mode du carnet de voyage. Sur la suggestion d'une collègue, nous avons ensuite opté pour l'expression «croquage de langue » terme multi-sensoriel et proche de la référence de départ au croquis, au carnet de voyage. Les caractéristiques du carnet sont débattues à l'oral puis rappelées dans la consigne écrite. Celle-ci reprend nos objectifs de formation en termes de didactique des langues : la réflexion sur la réception pluri-sensorielle des langues, sur les profils d'apprenants, en termes d'écoute, d'apprentissage, de mode d'expression, l'exploration de différents modes de biographie langagière et d'auto-évaluation. Les étudiants peuvent choisir une langue différente de celle pour laquelle ils se sont auto-évalués dans le Passeport des langues, une langue qu'ils aiment particulièrement ou au contraire qu'ils n'apprécient pas. 
Nous présentons et analysons les enquêtes sociolinguistiques et des collectes de langue faites par tous les étudiants, ainsi que les croquages de langue.

\section{2- Les cueillettes de langues et les enquêtes sociolinguistiques}

L'activité de collecte de langues amène les étudiants à faire une lecture fine de leur environnement urbain : entreprises, aéroports, gares, lieux touristiques, marchés, entrées d'immeubles, etc. Ils écoutent, observent, croisent les informations (codes sociaux et activité, éléments de spatialité et de temporalité). Ils explicitent par écrit leurs stratégies de collecte : ainsi un groupe préfère-t-il se concentrer sur les langues parlées dans les bus, où ils restent assez longtemps pour comprendre les situations de communication dont ils sont témoins.

La méthodologie d'enquête est choisie et les questionnaires élaborés par les étudiants. Ce sont eux qui déterminent la population enquêtée, souvent en lien avec le parcours de l'un des membres du groupe : par exemple, la communauté arménienne de la région lyonnaise, des parents d'élèves de collège. Un groupe décide de n'interroger que des étudiants, et confronte ses résultats avec les statistiques présentant le nombre et l'origine des étudiants étrangers inscrits dans l'enseignement supérieur ${ }^{2}$. Un autre conduit les entretiens avec chaque personne en plusieurs étapes « afin de pouvoir revenir sur ce qui avait été dit et, avec le recul suffisant, pouvoir aborder les questions plus en profondeur ». Plusieurs groupes s'intéressent aux apprentissages informels dans le cadre familial, et aux choix linguistiques des personnes en lien avec leur parcours de vie.

Les débats amènent plusieurs groupes à ne pas superposer nationalité, naissance et langue. Ils évitent la référence, dans leurs questions, à une supposée « langue d'origine », et à lui préférer des formulations liées au vécu des personnes : «Avez-vous de la famille autre que française?", "Quelles sont les langues avec lesquelles vous avez grandi ? ", "As-tu plusieurs langues maternelles, si oui lesquelles ?", "Quelles sont les langues que vous écoutez régulièrement dans votre environnement?"

La présentation par les différents groupes de leurs tâtonnements et de leurs choix, des éléments facilitateurs et des obstacles constitue l'un des temps forts de la formation. La lecture de leurs analyses nous éclaire également sur la réception des contenus théoriques apportés en cours, sur les besoins de formation qui persistent. Nous notons, en particulier, que les étudiants ne se contentent pas de lister les pratiques linguistiques : ils recueillent d'autres éléments, les stéréotypes sur différentes langues et cultures, le lien entre l'engagement associatif et la pratique des langues d'origine, l'influence des migrations interrégionales sur les usages linguistiques. Autant de témoignages de l'implication et de la réflexion 
sociolinguistique des étudiants.

\section{3- "Croqueurs de langue " et " non-croqueurs"}

Je compare pour cette étude les productions sociolinguistiques de mes étudiants, «croqueurs de langue » (88), et des étudiants de mes collègues, «non-croqueurs » (106), afin d'évaluer les impacts du croquage de langues sur les compétences plurilingues et sociodidactiques que nous souhaitons encourager. Il s'avère impossible de considérer la présence ou l'absence de croquage de langue comme seul déterminant des différences entre les deux groupes, tant d'autres éléments entrent en jeu. Tous ces éléments, en outre, ne sont pas accessibles: les références données en cours, la composition du groupe en termes de parcours universitaire antérieur, le poids donné par les formateurs à telle ou telle activité, etc. Ceci m'amène à mettre en regard, pour les deux groupes, les références bibliographiques, les items des entretiens ou questionnaires, l'analyse des enquêtes, et à ne considérer les différences comme signifiantes (en majuscules et centrés ci-dessous) uniquement si elles apparaissent pour au moins deux domaines.

C'est le cas pour la question interculturelle: les non-croqueurs sont proportionnellement plus nombreux que les croqueurs à citer des travaux sur l'interculturel, et à intégrer cette question à leur analyse finale. Les résultats quant à la question migratoire présentent des différences dans trois domaines, les références bibliographiques, les questions posées lors des entretiens et l'analyse finale : près de la moitié des croqueurs, contre un tiers environ des autres étudiants, ont fait référence à des travaux sur l'histoire de l'immigration; les croqueurs s'intéressent également plus souvent que les non-croqueurs à l'apprentissage et à la pratique de la langue du pays d'origine, à sa transmission aux enfants, au lien affectif à cette langue. Ils repèrent de nouveaux comportements, tels la fréquentation de forums, la correspondance électronique dans la langue d'origine. Ils intègrent à leur analyse finale une réflexion sur l'immigration et sur les liens entre plurilinguisme, l'apprentissage d'une langue 2 et l'apprentissage d'une nouvelle langue étrangère en milieu scolaire. Nous voyons par ailleurs, dans ces deux cas (l'interculturel, la migration), les interactions fructueuses entre la lecture de travaux de recherche, la mise en place d'observations fines et la densité de l'analyse. 


\begin{tabular}{|c|c|c|}
\hline \multicolumn{1}{|c|}{ Références bibliographiques } & 88 « croqueurs » & 106 « non-croqueurs » \\
\hline À L'HISTOIRE DE L'IMMIGRATION & 40, SOIT $45,46 \%$ & 30, SOIT $28,30 \%$ \\
\hline À la didactique des langues & 8, soit $9 \%$ & 16, soit $15,10 \%$ \\
\hline Aux travaux sur le plurilinguisme & 38, soit $43,18 \%$ & 72, soit $67,92 \%$ \\
\hline À DES PUBLICATIONS & 33, SOIT $37,5 \%$ & 56, SOIT $52,83 \%$ \\
\hline INSTITUTIONNELLES ${ }^{3}$ & & 27, soit $25,47 \%$ \\
\hline À la sociologie et à la socio & 4, soit $4,55 \%$ & \\
\hline
\end{tabular}

\begin{tabular}{|c|c|c|}
\hline Éléments pris en compte dans l'enquête & $88 \ll$ croqueurs $»$ & 106 «non-croqueurs » \\
\hline $\begin{array}{l}\text { LE PLURILINGUISME DANS LES } \\
\text { ESPACES PUBLICS }\end{array}$ & 64, SOIT $72,72 \%$ & 46, SOIT $43,40 \%$ \\
\hline $\begin{array}{l}\text { LE PLURILINGUISME DANS LES } \\
\text { ESPACES PROFESSIONNELS }\end{array}$ & 52, SOIT $59,09 \%$ & 74, SOIT $69,81 \%$ \\
\hline Le plurilinguisme dans les espaces privés & 71, soit $80,68 \%$ & 91, soit $85,85 \%$ \\
\hline $\begin{array}{l}\text { Les conditions d'immigration et } \\
\text { d'apprentissage du français }\end{array}$ & 16 , soit $18,18 \%$ & 19 , soit $17,93 \%$ \\
\hline $\begin{array}{l}\text { L'APPRENTISSAGE ET LA PRATIQUE DE } \\
\text { LA LANGUE DU PAYS D'ORIGINE }\end{array}$ & 69, SOIT $78,40 \%$ & 50, SOIT $47,17 \%$ \\
\hline $\begin{array}{c}\text { LA TRANSMISSION DE LA LANGUE DU } \\
\text { PAYS D’ORIGINE }\end{array}$ & 59, SOIT $67,05 \%$ & 46, SOIT $43,40 \%$ \\
\hline $\begin{array}{c}\text { LE LIEN AFFECTIF À LA LANGUE DU } \\
\text { PAYS D’ORIGINE }\end{array}$ & 38, SOIT $43,18 \%$ & 9, SOIT $8,49 \%$ \\
\hline $\begin{array}{c}\text { L'USAGE DES NOUVELLES } \\
\text { TECHNOLOGIES DANS LA LANGUE DU } \\
\text { PAYS D'ORIGINE }\end{array}$ & 4, SOIT 4,55 & 0 \\
\hline Les catégories socioprofessionnelles & 8 , soit $9,09 \%$ & 0 \\
\hline Les représentations sur le plurilinguisme & 58 , soit $65,90 \%$ & 86 , soit $81,14 \%$ \\
\hline
\end{tabular}




\begin{tabular}{|c|c|c|}
\hline Dans l'analyse de l'enquête, réflexion sur & 88 « croqueurs » & 106 « non-croqueurs » \\
\hline L'INTERCULTUREL & 21, SOIT $23,86 \%$ & 47 , SOIT $44,34 \%$ \\
\hline Les langues régionales & 34 , soit $38,64 \%$ & 33 , soit $31,13 \%$ \\
\hline L'IMMIGRATION EN FRANCE & 59, SOIT $67,04 \%$ & 63, SOIT $59,43 \%$ \\
\hline $\begin{array}{c}\text { LES LIENS ENTRE CONTEXTES } \\
\text { PLURILINGUES ET APPRENTISSAGES DE } \\
\text { LANGUES }\end{array}$ & 14 , SOIT 15,90 & 10 , SOIT $9,44 \%$ \\
\hline
\end{tabular}

\section{4- Les croquages de langue}

Les 79 croquages abordent 28 langues différentes : voici qui devrait nous rassurer quant aux ressources, parmi les étudiants qui se destinent à l'enseignement, pour mettre en place des activités d'éveil aux langues. Nous pouvons relativiser la prédominance de l'anglais, qui n'a été choisi que par 13 des étudiants pour leur croquage de langue.

Les étudiants n'ont pas tous mentionné la nature de leur lien à la langue abordée. Sur les 61 contextes précisés, les voyages en dehors du cadre scolaire (17 mentions) sont les plus nombreux, suivis des pratiques culturelles (14), des situations d'enseignement scolaire et universitaire et de jumelage (11), des liens familiaux (11), puis des séjours d'un an ou plus à l'étranger (4), des contextes professionnels (2) et des relations personnelles (2).

\section{1- Le plurisensoriel, les matériaux, les éléments représentés}

La consigne d'introduire les cinq sens est largement respectée. Elle induit de nombreuses correspondances entre les cinq sens et l'expression de la perception de la langue, de sa représentation, des vécus associés: parfums, paysages, souvenirs d'aliments de l'enfance, chants, instruments, toucher. Souvenirs et perception des sons sont représentés par de nombreuses matières et petits objets. Le toucher est largement convoqué : les langues sont lisses, douces, rêches, râpeuses comme les matériaux utilisés. L'odorat l'est aussi, de petits éléments sont enveloppés pour préserver leur parfum : lavande, vanille, cannelle, chocolat, poivre... La plupart des carnets sont riches en couleurs. Quelques productions ont recours à des clichés (par exemple, des couleurs chaudes pour l'espagnol), mais ce n'est pas systématique. Les correspondances rimbaldiennes sont largement présentes, et quelques étudiants les étendent aux résonances entre langue et autres productions culturelles : l'un d'entre eux associe l'architecture espagnole, celle des vestiges de l'histoire arabe comme celle de Barcelone, à la langue et à la musique : "la musicalité des courbes (rythme de la guitare espagnole), le mouvement et la danse des motifs et bas-reliefs (flamenco). Tout cela 
se retrouve dans la langue : le « $r$ » roulé, le glissé $d u$ « $s »$, le raclement $d u$ « $j »$, le rythme des phrases. " Pour une étudiante, le créole réunionnais est une langue qui « ressemble à son pays [...] en perpétuelle construction et même reconstruction, [...] révélatrice d'une culture française différente ».

Les symboles sont très nombreux et immédiatement compréhensibles (drapeaux, la croix occitane, cœurs) ou plus personnels. Les étudiants en donnent le sens : deux gants de boxe représentent «un combat lorsqu'il faut déchiffrer les mots de cette langue ». Un carnet est construit autour du double axe de l'ouverture et de la fermeture : la prison symbolise « la personne qui ne peut entendre et qui se retrouve au sein d'un groupe où personne n'a appris la langue des signes [...] le cadenas est emblématique de la situation et ne peut s'ouvrir que dès lors que nous possédons la bonne clé : l'ouverture d'esprit [...]».

Des objets sont insérés dans le carnet, collés, suspendus, accrochés, et pourraient faire l'objet d'un inventaire à la Prévert : clé, élastique, pince à linge, timbres-poste, aiguilles de pin, grains de riz, de coriandre, de cardamome... Les carnets comprennent aussi des inclusions de documents : plans de métro, cartes, extraits ou reproductions d'œuvres, photos de membres de la famille des étudiants, le plus souvent les grands-parents. La langue est directement incluse dans les carnets: partitions, citations d'auteurs, paroles de chansons, expressions (Jesus Tia), proverbes. Pour l'espagnol du Mexique, une étudiante inclut la chanson qui accompagne le jeu pour casser la piñata : «Dale, dale, dale, / No pierdas el tino. / Porque si lo pierdes / Pierdes el camino ॥. Ces extraits insérés sont autant de références à ce que l'auteur du carnet considère comme culture partagée non seulement avec ceux qui découvrent ce croquage de langue, mais aussi avec une large communauté culturelle.

\section{2- La mise en scène}

Les dispositions et mises en page sont complexes et diversifiées, les étudiants jouent avec les volumes et les surfaces : superpositions, jeux de transparence, découpages, tissus ou papiers froissés, graphies et calligraphies différentes. De nombreux croquages sont peints à l'aquarelle ou à la gouache, dessinés aux crayons de couleur ou pastels. L'un est une bande dessinée.

Lorsque les cartes géographiques ne sont pas la reproduction de cartes traditionnelles, elles sont recréées (le fleuve Gambie est un fil, Dakar une spirale de fil de fer) ou créées pour représenter les expériences vécues, à la manière des Cartes du Tendre. La Tamise est bordée de deux rives, celle des apprentissages (Learning) et celle des sentiments (Feeling). Un ruban trace le parcours de vie, de la «naissance d'une petite fille qui adore écouter [la langue de] ses grands-parents » au "jour [où] je l'espère, j'enseignerai cette langue », un autre est là 
pour «[...] faire part d'une langue qui partage ma vie grâce à mes grands-parents. C'est pour cela qu'à travers mon ruban [...] j'ai représenté ma courbe de vie. »

Des étudiants invitent à agir sur l'objet : il faut, pour le découvrir, ouvrir une boite ou des petites fenêtres, tourner un cadran, déplier; feuilleter un petit «cahier de voyage en France » pour découvrir l'anglais de France, ouvrir un cahier « conforme au modèle prescrit par la circulaire du ministre de l'instruction publique », vide jusqu'à son centre où est présentée la langue occitane. Certains objets sont enfermés dans des pochettes transparentes, certaines langues installées dans une enveloppe ; un sachet de tulle rose contient un caillou et du bois, il oscille sur un ruban entre deux petites pinces à linge.

\section{5- Les textes d'accompagnement}

Les commentaires des carnets introduisent la singularité de l'expérience, explicitent les différents éléments du croquage de langues. Nous analysons conjointement les textes inclus dans le carnet et ceux qui y sont ajoutés, au verso ou sur un feuillet séparé.

\section{1- Le non-linguistique de la langue}

Les croqueurs de langue évoquent leurs sensations auditives, en particulier pour des langues qu'ils connaissent peu : le son prime dans ce cas sur le linguistique : "J'ai choisi de réaliser un travail d'écoute de la langue italienne. C'est une langue que je ne connais pas, j'ai donc pu me consacrer exclusivement au ressenti et non à la signification de ce qui est dit. » Un étudiant établit une correspondance entre la couleur violette et le suisse allemand, pour lui « lingua incognita».

\section{2- Les éléments linguistiques}

Des aspects phonologiques des langues, les croqueurs représentent les sons spécifiques : des matériaux pour les [r] roulés de l'espagnol, des ondulations de rubans ou de la graphie d'une phrase pour la prosodie, pour le vietnamien, "langue monosyllabique tonale ». Une étudiante représente les accents toniques de l'italien par un graphique : les «intonations» en abscisse, "la parole» en ordonnée. Les étudiants expliquent leur représentation des sons de la langue choisie :

Cette langue m'évoque quelque chose d'entortillé, et à la fois des éclats de voix. [...] Le "papier mâché " représente cette sensation de non articulation et de non distinction des mots que l'on peut ressentir. [...] Un morceau de polystyrène car les tonalités de la langue ont un aspect qui me semblaient proches de cette sensation, avec une insistance sur certaines lettres, comme si on froissait notre phrase.

Sont abordées les familles de langue, les proximités (« Un papier transparent [...] 
pour marquer la transparence de la langue et donc les liens qu'il est possible de faire entre l'espagnol et d'autres langues (le français en particulier) »), ainsi les emprunts (les expressions latines présentes dans la langue française). Les étudiants mentionnent les variétés de langue, en particulier pour l'anglais, l'espagnol et le portugais, et la perception qu'ils en ont.

Un étudiant présente les contacts de langues, l'anglais et le français présents ensemble sur différents documents, ticket de cinéma, carte de restauration rapide. Plusieurs mentionnent les zones de diffusion de la langue. Un étudiant écrit à propos de l'anglais : «En ce qui concerne l'odorat j'ai trempé le bâton dans la vanille car c'est une plante que l'on peut glisser dans toutes les pâtisseries, ce qui me fait vraiment penser à cette langue qui plus tard sera surement parlée dans le monde entier. La vanille est partout, shampoing, savon, pâtisserie, parfum, tout le monde l'utilise. "

\section{3- Cultures et valeurs, données historiques}

Des éléments d'ordre culturel sont représentés, ainsi que les valeurs associées à la langue et à la culture ; celles-ci sont le plus souvent positives, car les étudiants ont choisi des langues qu'ils apprécient, à une exception près. Lorsqu'elles sont négatives, c'est en référence à l'histoire. Dans un carnet à propos de l'espagnol, Mafalda ${ }^{4}$ fait face à Castro. Dans un autre, sont présentés des poèmes de Jara et de Neruda : "J'ai toujours baigné dans cette culture, culture de lutte et de sang, d'espoir et de courage, de joie de vivre et de poésie. » Une étudiante commente son aquarelle à propos du flamand : "La coupure en deux par une ligne continue, non volontaire au moment de la création, n'est peut-être pas étrangère au conflit de culture et d'identité qui habite ce pays [la Belgique]. » Je reçois le carnet d'un étudiant: page de brume où le soleil tente de percer, pliée en accordéon; des gouttes bleues sont fixées de manière instable par des punaises. Je m'étonne, pourrait-on les fixer à l'aide de liège ou de pâte ? «Non, me répond-il, ça pique et ça se défait, c'est du bosniaque. »

\section{4- Modes d'apprentissage, stratégies}

Une étudiante évoque les étapes successives de l'écoute, de plus en plus fine, à propos du lao : "Les bouts de tissus à forme géométrique que j'ai collés. [Dans un] premier temps il semble que tous les bouts de tissus sont identiques, mais lorsque on s'habitue et qu'on les observe bien on remarque et on distingue les différences. » Une autre évoque ses difficultés avec le [th] anglais "cette petite mouche [...] la bête noire de cette langue. »

Les étudiants mentionnent les circonstances de leurs apprentissages, dans le cadre scolaire ou non. Une étudiante aborde son apprentissage informel du turc, et sa mise en page 
évoque aussi son lien à la France: "En bas à droite, un article de journal que j'ai fait "fusionner" avec la feuille. Je n'ai jamais pris de cours de turc, et j'ai appris à lire cette langue toute seule assez tôt grâce aux journaux de mon grand-père. Le papier journal a fusionné avec mon support pour représenter ma propre intégration. » Une étudiante traduit en vers son sentiment de désorientation, ses stratégies non verbales, son recours à la lecture, à propos de l'anglais.

\section{5- Les expériences de vie}

Les étudiants relatent les vécus associés à la langue abordée, voyages, rencontre amoureuse, "Love and autres drogues », séjours longs, amitiés. Les vécus familiaux sont très présents («Mon grand-père qui m'a tout appris», photographié devant une usine) et indéfectiblement liés à la perception de la langue : " on venait vous voir toi et papy, [...] l'odeur de ta sauce tomate embaumait tout l'immeuble et vous nous appreniez quelques mots. » $\mathrm{Ou}:$ : Le créole [guyanais] m'évoque également l'interdit et notamment le fait que les enfants ne devaient pas l'employer en présence d'adultes. »

\section{6- Des éléments de biographie langagière et didactique}

Ces croquages illustrent l'ancrage des apprentissages sur les expériences personnelles et les sensations, les liens forts entre biographie langagière et «biographie didactique» (Mercier). La temporalité des apprentissages, comme l'a analysé A. Mercier pour les mathématiques, ne se réduit pas à celle de la salle de classe : «Le temps didactique est, pour l'élève, une loi de l'institution didactique, car le temps produit par la succession des objets du savoir enseigné n'est pas le temps de la biographie didactique, le temps que rythment les ignorances que l'élève rencontre, puis dépasse : c'est donc une fiction fonctionnelle » (53).

Mercier montre dans sa thèse comment l'accès à un épisode passé de la biographie didactique de l'élève permet «d'entreprendre la reconstruction d'un rapport à ces objets de savoir - «premiers », mais inconnus à priori de l'enseignante comme des étudiants euxmêmes - qui rendra possible la poursuite de la construction biographique d'un rapport personnel [aux savoirs enseignés] » (212). Nous voyons, dans les croquages qui traitent de langues enseignées en milieu institutionnel, la part des savoirs acquis hors du temps scolaire ou universitaire. Que ce soit dans ces croquages ou dans ceux sur les langues fréquentées en dehors des lieux d'enseignement, la réflexion métalinguistique, métaculturelle et métacognitive constitue une matière où puiser ce qui relève des compétences transférables d'une langue à une autre, de l'environnement scolaire à d'autres environnements, et vice versa. 


\section{6- De nouvelles perspectives}

Ces activités d'évaluation ont suscité une réflexion sociolinguistique et métalinguistique, ont encouragé une lecture fine par les étudiants de leur environnement, geste précieux pour de futurs enseignants. Les croquages de langues ont été fortement investis : en témoignent la variété des productions et des modes d'expression, le soin apporté à leur réalisation. Ils présentent l'intimité de la relation à une langue, qu'il s'agisse des perceptions de la langue elle-même ou des expériences qui y sont liées, ils valorisent les référents culturels partagés ou susceptibles de l'être : les tickets de bus, par exemple, rendent compte d'un vécu personnel tout en étant immédiatement compréhensibles par tous. Ces croquages donnent à voir plusieurs espaces, et les explorer nous amène à découvrir où leurs auteurs ont choisi de poser leur curseur, sur les lignes qui vont du très intime au très connu, à ce qui dans la langue-culture est largement publié et arpenté par tous ; du discours littéraire aux expressions familières; de la perception des sons d'une langue à l'écriture.

A propos des langues des croquages, nous pouvons reprendre ce qu'écrit Valentin N. Voloshinov du mot: [aucune langue] «n'est donné[e] à l'artiste dans une sorte de virginité linguistique. [La langue] est déjà fécondé[e] par les situations vécues et par les contextes poétiques où [elle] a été rencontrée » (cité dans Todorov 275). Encouragés à rendre compte de leurs perceptions et de leurs expériences d'une langue, les auteurs des carnets ont tissé ensemble l'individuel et le collectif, les découvertes personnelles et ce qui a été donné en partage.

L'architecture du module de formation n'a pas permis de retour sur le temps de la formation, d'analyse des productions, de mise en perspective pour la classe. Ce temps serait précieux pour mutualiser les apports en termes de réflexion métalinguistique, interculturelle et sociolinguistique, analyser les récurrences dans les modes de représentation (le recours aux citations, par exemple, comme interaction avec une communauté large et invisible dont nous nous sentons membres), expliciter ces espaces féconds de langue que sont les démarches créatives, et en extraire des modalités d'enseignement, envisager des activités plurisensorielles liées à l'enseignement des langues.

Les étudiants utilisent les matériaux simples de leur environnement proche et privilégient la perception tactile. Construits à partir « d'éléments incomposables et d'images perdues venues du quotidien et de la nature » (Germano Celant, 1967, à propos de 1'Arte Povera), les croquages de langue sont des compositions, des re-compositions qui peuvent servir de point de départ à une réflexion sur l'Arte Povera des années 1970, ainsi que sur Art\&Language. A l'instar des œuvres d'art conceptuel, sont présents dans ces croquages de 
langue de nombreux supports, validés en tant qu'ils réfèrent à la langue : cartes, partitions, objets... Sont mises en scène plusieurs représentations d'un même objet-langue, ou d'un même concept associé à cette langue. Il ne s'agit pas de répétitions, mais de différentes solutions pour relayer, par un mode de représentation, ce qu'un autre mode ne réussit pas à exprimer tout à fait : ainsi de la prosodie exprimée par l'ondulation de la mise en page, du concept de tradition, présent par le mot lui-même, et renforcé par l'effet parchemin, etc. Ces processus peuvent être comparés à celui de Joseph Kosuth pour One and Three Chairs: l'objet réel, sa photographie et l'affichage de sa définition dans un dictionnaire sont les trois éléments convoqués pour rendre compte du concept de "chaise ». La représentation de la langue elfique écoutée dans les films Le Seigneur des Anneaux, et le texte qui l'accompagne, en sont une illustration, particulièrement à propos des sons perçus comme « métalliques » :

La première sensation qu'on a en écoutant cette langue est indéniablement son aspect éthéré, doux. En essayant de l'expliquer, je n'ai trouvé qu'une seule image : C'est comme entendre les feuilles tomber : douceur, légèreté ». [...] A cela s'ajoutait l'impression d'une langue usée, vieillie, soufflée, voir exhalée: la corde usée me donne cette impression de vieillesse. [...] j'ai été intrigué par certaines prononciations, dans différentes situations. Ce souffle qu'est la langue est entrecoupé de lettres tranchantes, de phonèmes durs. Après avoir remarqué ceci, cet aspect tranchant et métallique se trouvait sous-jacent à toute l'énonciation elfique : j'ai tenté de le représenter en structure, le métal qui sous-tend tout le reste [...].

Un même concept peut être représenté plusieurs fois (une étudiante double son texte sur le bressan, "c'est la tradition ", d'une présentation de son carnet comme un parchemin, brun et brulé sur ses bordures), ou un même élément peut rendre compte de plusieurs perceptions et idées: "J'ai voulu transcrire la rugosité de la langue, comme la "jota» à travers une inscription en calligraphie arabe (" espagnol»). Cela rend compte à la fois d'une perception sensorielle mais aussi de l'histoire du pays et de son lexique qui emprunte de nombreux mots à la langue arabe ». Les éléments de représentation, textes, documents écrits, matières, travail sur la matière (peinture en couche épaisse ou travaillée en aquarelle, tracés de crayon ou remplissage de surfaces, découpages et superpositions...) sont en dialogue les uns avec les autres; les modes choisis pondèrent ou au contraire accentuent certains éléments, et cette intertextualité constitue en elle-même un discours.

L'activité de croquage de langues, associée à l'enquête sociolinguistique, a permis aux étudiants de percevoir le plurilinguisme « comme une réalité proche et palpable, loin des représentations circulantes renvoyant le plus souvent à un objet exotique et extraordinaire» (Castellotti et Moore 62). Elle a contribué à leur réflexion sur leurs biographies langagières, 
et a donné la part-belle aux «sensibilités linguistiques »(Glissant 27). Elle les a placés, comme le souhaite Joëlle Aden à propos du lien entre pratiques artistiques et langagières, moins « du côté des récepteurs-commentateurs », plus «du côté des créateurs-interprètes » (9), et ce côté-là, nous le voyons, génère une réflexion précieuse pour le futur enseignant. Les étudiants témoignent de leur réflexion sur le plurilinguisme : "Doit-on alors parler vraiment de langues "étrangères", si ces dernières font parties de notre vie quotidienne? " ou encore: "On peut dire que les langues n'ont pas de frontières. Elles voyagent avec les individus qui les parlent $»$. Plus que des objets de médiation entre les étudiants et leur environnement linguistique, ces croquages de langue s'avèrent des ouvrages de médiation, des témoignages du processus de recomposition de leurs paysages linguistiques par les étudiants, de développement de leurs compétences plurilingues.

Un groupe d'étudiants crée un mot pour désigner ces horizons nouveaux, qui sera notre conclusion :

Etre plurilinguiste ${ }^{5}$ donnerait de la confiance, de l'épanouissement mais aussi et surtout une compréhension plus fine de certains élèves. Finalement, il y aurait ou devrait y avoir une prise de conscience de cette évolution des pratiques langagières $d u$ fait de la multiplicité des origines, cultures, ... de chacun. Nous pouvons conclure, grâce à cette enquête, que l'école devrait garantir à chaque famille la reconnaissance et la valorisation de la culture de chacun de ses élèves afin de soutenir le respect et l'harmonisation du vivre ensemble.

\section{Bibliographie}

Aden, Joëlle. Introduction. Delahousse, B. et M.-P. Hamez. Pratiques artistiques et pratiques langagières : quelle synergie? Les Langues Modernes 2 (2010) : 9-13.

Akinci, Mehmet-Ali. «France multilingue : richesse ou danger? Résultats d'une enquête dans les écoles élémentaires de Lyon ». Écarts d'identité (2003) : 41-46.

Beckett, Samuel. En attendant Godot, Paris : Minuit, 1952. Acte II. 87-88.

Castellotti, Véronique et Danièle Moore. «Parcours d'expériences plurilingues et conscience réflexive ». Biographie langagière et apprentissage plurilingue, Le français dans le monde numéro spécial. Dir. Muriel Molinié. Paris : Clé international, 2006. 54-68.

Dinvaut, Annemarie. " La didactique plurilingue des langues : obstacles et propositions ». Thèse de doctorat, 2008.

http://demeter.univ-lyon2.fr/sdx/theses/lyon2/2008/dinvaut_a

Glissant, Edouard. Entretiens avec Lise Gauvin. L'imaginaire des langues. Paris : Gallimard, 2010.

Mercier, Alain. «L'élève et les contraintes temporelles de l'enseignement, un cas en calcul 
algébrique ». Thèse de doctorat, 1992.

halshs.archives-ouvertes.fr/docs/00/27/82/99/PDF/These_Mercier_Alain.pdf

Perea, Encarnaciòn Carrasco et Enrica Piccardo. "Plurilinguisme, cultures et identités ». Altérité et formation d'enseignants. Dir. Marinette Matthey et Diana-Lee Simon, Lidil 39. Grenoble : Ellug, 2009.

Voloshinov, Valentin N. «Les frontières entre poétique et linguistique ». Todorov, Tzvetan, Mikhaïl Bakhtine, Le principe dialogique, suivi de Écrits du Cercle de Bakhtine. Paris : Seuil, 1981.

\section{NOTES}

${ }^{1} \mathrm{http}: / /$ www.europe-education-formation.fr/europass-langues.php

${ }^{2}$ Repères et références statistiques, édition 2010, visible sur le site : http://media.education.gouv.fr/file/2010/16/9/RERS_2010_152169.pdf p.192

${ }^{3}$ Ministère de l'éducation, sites de l'union européenne, du Conseil de l'Europe.

${ }^{4}$ Personnage principal de la bande dessinée à caractère politique de Quino, Argentin.

${ }^{5}$ C'est nous qui soulignons. 